\title{
Linguistische Analysepraxis
}

\author{
Heidrun Kämper
}

\section{Zeitreflexion und Sprachgeschichte}

\section{Ein Konzept zur Erforschung des Nachkriegsdeutschen}

\section{Vorbemerkung}

Dieses Buch wendet sich an alle, die Interesse an sprachlich interpretierter Zeitgeschichte haben - an Historiographen, Politologen, Soziologen, an Lehrer, Journalisten und interessierte Laien und auch an Linguisten, vorzugsweise an Sprachhistoriker. Methodisch einzuordnen ist es als ein Beitrag zu einer kulturgeschichtlich ausgerichteten und diskursiv angelegten Sprachgeschichte des 20. Jahrhunderts, die sprachliche Daten so aufbereitet, dass sie z.B. mentalitätsgeschichtlich interpretierbar sind -

so etwa könnte die Einleitung eines Buches lauten, das noch nicht geschrieben ist. Es soll aus einem Vorhaben hervorgehen, von dem ich insofern berichte, als die folgenden Überlegungen die methodische Positionierung dieses Projekts wiedergeben. ${ }^{1}$

Die uns von der Veranstalterin und den Veranstaltern dieser Tagung nahe gebrachte Leitidee einer der Öffentlichkeit zugänglichen, ihrer gesellschaftlichen "Bringschuld « nachkommenden »Sprachverhaltenslinguistik ${ }^{2}$ ist die

1 Das Projekt »Zeitreflexion im ersten Nachkriegsjahrzehnt. Ein Beitrag zur Sprachgeschichte 1945 bis 1955« wird seit April 2000 (und bis Marz 2002) von der deutschen Forschungsgemeinschaft gefördert und von zwei wissenschaftlichen Mitarbeitern und mir am Institut für Deutsche Sprache, Mannheim, realisiert.

2 Ich verweise auf Ortner/Sitta (in diesem Band: 8), die >Bringschuld، als Verpflichtung der Wissenschaft verstehen, die Fragen zu beantworten, »die Menschen (außerhalb der Wissenschaft) bewegen und für deren Beantwortung die Gesellschaft Menschen unter (im Ganzen immer noch) komfortablen Bedingungen freistellt, angehen.« In dem Konzept einer Sprachverhaltenslinguistik ist diese Verpflichtung aufgehoben. Eine solche Linguistik, die »nahe am sprechenden/ schreibenden Menschen bleibt« (ebd.: 61) und deren Sprachbegriff nicht nur den 
Richtmarke, an der sich diese einleitenden Formulierungen orientieren und die ich im Folgenden thesenhaft kommentiere. Dieser Kommentar bezieht sich auf das Selbstverständnis der Linguistik als kulturwissenschaftliche Disziplin sowie auf zwei interdisziplinäre Ansätze von besonderer Forschungsdynamik - der Diskurs- und Mentalitätsanalyse. Sie scheinen für die Linguistik resp. Sprachgeschichte einige Brisanz zu haben.

\section{Linguistisches Selbstverständnis: Kulturwissenschaft}

Kulturwissenschaft ist eine Superdisziplin, der sich eine Reihe von Einzeldisziplinen subordiniert haben - eine davon ist die Sprachwissenschaft. Peter Auer (in diesem Band) hat in seinem Beitrag diesbezüglich argumentiert. Insofern sich kulturwissenschaftliche Linguistik in die Nachbarschaft anderer kulturwissenschaftlicher Disziplinen begibt, scheint eine methodologische Reflexion, die Abgrenzung und auch und nicht zuletzt Legitimierung zum Ziel hat, unausweichlich.

Kultur ist »das >Ganze des wechselseitigen Zusammenspiels von Denkformen, Formen des Sich-Verhaltens und sozialen Handelns und den [...] daraus entstehenden Objektivationen « (Oexle 1996: 26). Indem also Objektivationen sozialen kulturellen Handelns den Gegenstand der kulturwissenschaftlichen Disziplinen bilden und indem Sprache eine solche Objektivation ist, ist Sprachwissenschaft eine Kulturwissenschaft, die sich mit der Beschreibung von Sprache als Form sozialen kulturellen Handelns beschäftigt. Sprachgeschichte stellt sich mithin dar als eine Sektorwissenschaft der Superdisziplin Kulturgeschichte. Dieses Selbstverständnis der Sprachwissenschaft resp. Sprachgeschichte ist nicht neu. Hermann Paul hat es bereits in den >Prinzipien formuliert. Es ist heute erweitert um die Vorstellung der sprachlichen Konstituierung von Wirklichkeit. Sprache und Kultur, Sprachwissenschaft und Kulturwissenschaft treffen sich in dem Konzept von der gesellschaftlichen Konstituierung von Wirklichkeit. Diese für die kulturwissenschaftlichen Disziplinen bekanntlich approbierte These von der "gesellschaftlichen Konstruktion von Wirklichkeit« (so der Titel von Berger/Luckmann) heißt: In Sprache sind Deutungsschemata vorgeprägt, mit welchen Wirklichkeit interpretiert wird. Kultur ist auf Kommunikation angewiesen und wird durch

des sprachlichen Systems umfasst, interessiert »sich für individuelles Sprachverhalten, seine Entstehung und seine systematischen Entwicklungsmöglichkeiten $[\ldots]$ ebenso [...] wie für kollektives [Sprachverhalten]«, um Sprache auch »als Medium des kollektiven Gedächtnisses einer Gesellschaft « (ebd.: 13) zu beschreiben. 
Sprache vermittelt und konstituiert in der Funktion von $»$ Wirklichkeitsdeutung " (Hansen 1999: 391). Sprachgeschichte fragt in diesem Sinn nach den historischen Bedingungen und Veränderungen von Sprache als Vermittlerin zwischen Mensch und Wirklichkeit.

Mit dem Selbstverständnis von Linguistik als Kulturwissenschaft ist der Grundton ihrer erkenntnisleitenden Fragestellungen angestimmt. Die Herausforderung heute besteht besonders für die Sprachgeschichte nicht nur in Methodenpluralität und reklamierter Operationalisierbarkeit, sondern auch in der Auseinandersetzung mit Analyseansätzen, die sie mit kulturwissenschaftlich orientierten Nachbardisziplinen, vor allem mit der der Historiographie, teilt. ${ }^{3}$ Diese wissenschaftstheoretisch bedingte Entdisziplinierung der Analyseansätze erfordert gleichzeitige Disziplinierung ihrer fachbezogenen Ausformung. ${ }^{4}$ Zwei dieser Ansätze - Diskursanalyse und Mentalitätsanalyse möchte ich vor dem Hintergrund des Leitgedankens >Entdisziplinierung und Disziplinierung `diskutieren.

\section{Linguistische Diskursanalyse fragt: Wie und warum?}

Diskursanalytisch ${ }^{5}$ orientierte Sprachgeschichte überschreitet Grenzen. Was z.B. Historiker als Voraussetzung für ihre Erkenntnis formulieren - die

3 Insofern scheint auf die heutige Linguistik mutatis mutandis zuzutreffen, was Beaugrande (1997: 6) für ihre fruhen Jahre beschreibt, da sie »mit Recht [furchtete], von den Nachbarwissenschaften vereinnahmt zu werden «. Das daraus entstandene Anliegen, »eine Wissenschaft sein [zu wollen], die nur >Sprache an sich ergrunden sollte , kann allerdings wohl als obsolet betrachtet werden. Vereinnahmung einerseits, Abgrenzung andererseits sind heute weniger Angstkategorien als vielmehr Anlässe, sich der disziplinären Leistungsfähigkeit zu vergewissern, ohne Exklusivităt des Gegenstands zu beanspruchen.

4 Oexle (1996: 31) stellt fest, dass die "Geschichtswissenschaft [...] [einer] entschlossenen Entdisziplinierung [bedarf], was vielleicht auch fur andere kulturwissenschaftliche Fächer gilt«. Gleichzeitig ist das Gegengewicht einer Disziplinierung gefordert min dem Sinne [...], daß die einzelnen kulturwissenschaftlichen Fächer sich ihrer historisch gewordenen, spezifischen Leistungen bewußt sind und sie zur Geltung bringen«.

5 Hier soll nicht der in der (Sprach-)Historiographie gefuhrte >Diskurs «-Diskurs rekapituliert und bewertet werden, der sich u.a. durch die Klärung des einerseits bereits von der Gesprächsanalyse besetzten (vgl. etwa Ehlich 1994), andererseits aus dem französischen Konstruktivismus (Foucault) stammenden Terminus auszeichnet. Vgl. u.a. Busse/Teubert (1994) und Mainguenau (1994). 
kommunikationsgeschichtliche Fundierung -, ist zugleich (neben anderen) originäres Geschäft einer diskursiv angelegten Sprachgeschichte. Die diskursive Anlage von sprachanalytischen Vorhaben setzt Serien von in thematischer Beziehung zueinander stehenden Texten voraus. In diesem Konzept der Serie ist das kulturgeschichtliche Merkmal 'kollektiv s aufgehoben. ${ }^{6}$

Sprachwissenschaftliche Diskursanalyse fragt danach, wie und warum ein Sachverhalt, ein Ereignis, eine Befindlichkeit von Zeitgenossen einer bestimmten Epoche oder einer bestimmten gesellschaftlichen Formation in Sprache gefasst wird. So ist Diskursanalyse zu verstehen als Instrumentarium, größere Datenmengen nach inhaltlichen Kriterien zu strukturieren - als Hilfskonstruktion mithin zur Erlangung sprachlicher Erkenntnisse, welche eine Sprachgemeinschaft, ein Denkkollektiv ${ }^{7}$ betreffen.

Uns interessiert etwa: Wie sprechen Elite-Formationen der Besatzungszonen bzw. der Bundesrepublik und der DDR - nämlich Politiker, Juristen, Theologen, Wissenschaftler/Philosophen und Künstler -, wie sprechen Täter und Opfer in den Jahren 1945 bis 1955 über die jüngst vergangenen zwölf Jahre, über ihre Gegenwart und über ihre Zukunft? Diese drei Zeitorientierungen >Vergangenheit ‘, , Gegenwart‘, 'Zukunft Superdiskursen. Ihnen zugeordnet sind je spezifische Subdiskurse - den Superdiskurs >Vergangenheit etwa konstituieren Subdiskurse wie >Ursprünge des Nationalsozialismus‘, ’Erscheinungsformen des Nationalsozialismus ‘, ১Schuld৫. Ein typischer Gegenwartsdiskurs trägt den Titel ’Sieger und Besiegte`, ein typischer Zukunftsdiskurs heißt >Die neue Ethik`. Und indem diese Zeitreflexionen der argumentativen Konstituierung einer deutschen Identität dienen, fragen wir weiter: Mit welchen Merkmalen und Bewertungen werden die Themen versehen, welche die drei Zeitdimensionen konstituieren, um zu dieser intendierten Identität zu gelangen?

Diskurse sind überindividuelle sprachliche Erscheinungen. Auf Diskursebene werden Themen beschrieben - auch von Nichtlinguisten, von Historikern etwa. In wissenschaftstheoretischer Hinsicht ist also der Untersuchungsgegenstand >Diskurs، eine Schnittstelle der kulturwissenschaftlichen Disziplinen - hier findet 'Entdisziplinierung، statt. Die geforderte gleichzeitige >Disziplinierung، heißt für die Linguistik als Kulturwissenschaft: diskursive

6 Die moderne Kulturwissenschaft ist sich [...] einig [...], daß die Gegenstăndlichkeit von Kultur als kollektive Geistigkeit bestimmt werden muß« (Hansen 1999: 247).

7 Wir folgen mit dieser glưcklichen Bezeichnung Ludwik Fleck, der >Denkkollektiv c definiert als »Gemeinschaft der Menschen, die im Gedankenaustausch oder in gedanklicher Wechselwirkung stehen «. So verstanden $»$ besitzen wir in ihm den Träger geschichtlicher Entwicklung eines Denkgebietes, eines bestimmten Wissensbestandes und Kulturstandes, also eines besonderen Denkstiles« (Fleck 1980: 54f.). 
Serien derart zu parzellieren, dass eine Beschreibungsebene erreicht wird, die es der Linguistik gleichsam erlaubt, zu sich selbst zu kommen. Linguistik, die bei sich selbst ist, fragt nach dem $>$ Wie $<$ und nach dem $>$ Warum $<$ des sprachlichen Ausdrucks.

Peter Auer hat kulturwissenschaftliche Linguistik als »Differenzwissenschaft « beschrieben und reklamiert das >Wie als einen ihrer zentralen Gegenstände (Auer 2000: 67f.). Dieses >Wie $<$ wird greifbar in der diskursiven Serie - als Ausdrucks- und Bezeichnungskonkurrenzen und -alternativen z.B., für die verschiedene Namen existieren: Mit der Beschreibung von Schlüsselwörtern oder Leitvokabeln etwa wird sozusagen das diskursive Gerinnungsprodukt dargestellt. Die Arbeiten von Stötzel/Wengeler (1995) sind hier exemplarisch. Wer >Ideen` bzw. >Vorstellungen` beschreibt - Fritz Hermanns (demnächst) hat jüngst für die Bewillkommnung dieser wiedergekehrten Kategorien plädiert -, den interessiert ein Fond von durch den sprachlichen Ausdruck mitbezeichneten Denkhorizonten. Das kognitionswissenschaftlich begründete Konzeptmodell (vgl. etwa Fraas 1996 und Konerding 1993) schließlich erlaubt es, sprachlichen Ausdruck zu betrachten, um seine Komplexität, Strukturiertheit und sein (historisches, gesellschaftliches) Geprägtsein darzustellen.

Für welche dieser Kategorien man sich auch entscheidet - insofern Wirklichkeit diskursiv erzeugt wird und insofern Schlüsselwörter oder Ideen oder Konzepte in Diskursen sprachlich realisiert und damit manifest werden, sind sie Träger der diskursiv erzeugten Wirklichkeit. Sie sind als explizite sprachliche Realisierungen von Wissensbeständen in Form von Kontextualisierungen aus Texten zu erschließen und im Sinn kollektiver Deutungen beschreibbar. Eine solche Vielzahl sprachlicher Ausdrücke zur Erfassung und Konstituierung eines bestimmten Wirklichkeitsausschnitts ist im Sinn etwa von Josef Kleins (1989: 17ff.) Bezeichnungskonkurrenz (mehrere Bezeichnungen derselben Sache) und Bedeutungskonkurrenz (mehrere Bedeutungen desselben Ausdrucks) darzustellen.

Es ist ein Unterschied, ob eine gesellschaftliche Formation sich als Opfer oder als Täter konzeptualisiert, ob sie von ihrer Schuld oder Verantwortung oder von ihrem Irrtum spricht. Es ist ein Unterschied, ob ein Theologe oder ein Kommunist von Schuld spricht. Es ist ein Unterschied, ob ein ehemaliger jüdischer KZ-Gefangener oder ein Mitläufer oder ein zu zwanzig Jahren Zuchthaus verurteilter NS-Täter sich als Opfer bezeichnet. Es ist ein Unterschied, ob im Mai 1945 oder im Oktober 1953 Schuld sprachlich erfasst wird. Und da die sprachliche Konstituierung von Wirklichkeit auch mit Häufigkeit und Intensität zu tun hat (vgl. Berger/Luckmann 1999: 165), hängt sie auch davon ab, wie oft und wie eindringlich man von sich als Opfer bzw. Täter, von Schuld, Verantwortung oder Irrtum spricht - die diskursive Serie bietet für dieses Erkenntnismoment die Grundlage. 
Indem Sprachwissenschaft nach dem $>$ Warum , fragt, legt sie Funktionen sprachlicher Äußerungen offen. Diese Funktionen lassen sich im Sinn von Argumentationsstrukturen beschreiben. ${ }^{8}$ Die Diskurse der frühen Nachkriegszeit bilden einen Argumentationskomplex. Die Argumente werden von einer Argumentationsgemeinschaft, der Funktions- und Interpretationselite, vorgetragen, deren Angehörige (die meisten, jedenfalls diejenigen, die in unserem Textkorpus vertreten sind) ein übergeordnetes Argumentationsziel haben: die Rehabilitierung der Deutschen und ihre Wiedereingliederung in die Demokratien der Welt. Dieses generelle Argumentationsziel hat einen Anlass, den Vorwurf nämlich, den die Alliierten erheben und der im Sinn eines Geltungsanspruchs lautet: >Die Deutschen sind ein verachtenswertes Volk. Sie missachten grundlegende Werte, sind Nazis allesamt . Dieser Vorwurf stellt, wenn wir die Nachkriegsdiskurse der deutschen Funktions- und Interpretationselite auf einer Argumentationsstruktur abbilden, den strittigen Fall dar, die die Argumentation auslösende Quaestio. ${ }^{9}$ Diese Quaestio schafft für die deutsche Elite eine "Problemlage« (Kopperschmidt 1989: 58). Denn ihre Sichtweise auf die Deutschen ist eine andere, und ihrem eigenen Selbstbild folgend ${ }^{10}$ widersprechen sie dieser Quaestio, indem sie mit der These -Die Deutschen sind ein achtenswertes Volk، den entgegengesetzten Geltungsanspruch erheben. Damit haben sie die Beweislast, soll heißen: Sie müssen durch die Formulierung zustimmungsfähiger, überzeugungskräftiger, unstrittiger, als geeignet anerkannter und relevanter Geltungsgründe, m.a.W. durch plausible und akzeptable Argumente die Welt überzeugen. Diese Geltungsgründe bringen sie als je spezifische Exemplifizierungen des generellen Geltungsanspruchs innerhalb verschiedener Diskurse der Nachkriegszeit vor, z.B., wenn sie über das sehr dominante Thema >Schuld ‘ reden, über das >OstWest $<-$ Problem, wenn sie sich über die ,Welt, in der wir leben $<$ verständigen

8 Folgendem liegen u.a. die Arbeiten von Klein (bes. 2000), Kienpointner (1992) und Kopperschmidt (1989) zugrunde. Kopperschmidt definiert Argument als „Funktionskategorie, die [...] die Rolle einer Äußerung als Geltungsgrund furr den problematisierten Geltungsanspruch einer anderen Äußerung kennzeichnet* (1989: 95).

9 Vgl. etwa Kopperschmidt (1989: 54): ")strittig، ist eine mögliche Eigenschaft von Geltungsanspruchen, insofern uber deren Berechtigung zwischen kommunizierenden Subjekten ein Dissens besteht $\kappa$.

10 Das Selbstbild der >geistigen Elite in der fruhen Nachkriegszeit, derjenigen also, die offentlich reflektieren und Verantwortung nicht nur fuhlen, sondern vielfach auch nach dem 8. Mai 1945 ubernehmen, ist geprägt von Kompetenzanspruch und einem daraus abgeleiteten Recht zu Korrektur und Wegweisung. Entsprechend ist ihr Argumentationsverhalten beschaffen (vgl. zum gruppenspezifischen Argumentationsverhalten Kopperschmidt 1989: 175; zur diskursiven Spezifik von Topos-Konfigurationen Klein 2000). 
und über die `Zeit, in der wir leben`. Der Diskurs, der dieses Gegenwartsbewusstsein etwa der Nachkriegseliten erschließt, ist ein argumentationslogischer Komplex aus Konzession, Geltungsanspruch, Konklusion. Konzession meint: Man redet von einem deutschen Ende, vom Ende der deutschen Geschichte, vom Nichts, vor dem die Deutschen stehen. Dennoch: Das Gegenwartsbewusstsein der geistigen Elite im Nachkriegsdeutschland ist nicht wie gelegentlich behauptet - gekennzeichnet von einem Endzeit-Gefühl, von Orientierungslosigkeit. Vielmehr sind Äußerungen, die das Ende Deutschlands verkünden, argumentationslogisch $\mathrm{zu}$ interpretieren als Konzessionen, die man angesichts der unübersehbaren desolaten Nachkriegswirklichkeit macht, die aber hinsichtlich des Geltungsanspruchs dieses Subdiskurses nämlich die Propagierung einer neuen Chance - keine Bedeutung haben. >Die Deutschen haben eine neue Chance ist der Geltungsanspruch und die Exemplifizierung des generellen Geltungsanspruchs , Die Deutschen sind ein achtenswertes Volkr. Dieses Argument sucht man den Zeitgenossen nahe zu bringen, und man stützt es, indem man - argumentationslogisch im Sinn einer Konklusion - Forderungen der Stunde formuliert: >Wir haben eine neue Chance, also lautet die Forderung der Stunde: Lösung unserer Aufgaben!

Wir sehen: ‘Diskursanalyse meint nicht einen methodischen Ansatz, sondern ist Bezeichnung für die Herangehensweise an eine spezifisch beschaffene empirische Basis, nämlich die Serie thematisch kongruenter sprachlicher Äußerungen. Diskursanalyse stellt die Voraussetzung dar für methodische Ansätze, die für die Sprachwissenschaft die Fragen nach dem $>$ Wie $<$ und dem ,Warum، sprachlicher Erfassungen durch ein Kollektiv beantworten. Wir haben mit der Extraktion von Schlüsselwörtern und der Rekonstruktion von Argumenten Beispiele für mögliche Antworten angedeutet.

\section{Linguistische Mentalitätsgeschichte ist Mentalitätsbewusstseinsgeschichte}

Die zweite Konzeption, um welche sich kulturwissenschaftliche Disziplinen heute scharen und welche es für die Sprachgeschichte zu spezifizieren gilt, firmiert unter dem Namen >Mentalitätsgeschichteく. Wir rekonstruieren Zeitreflexionen als dominierendes Denk- und Deutungsmuster der Funktions- und Interpretationselite in der frühen Nachkriegszeit im Sinn von Haltungen, Einstellungen, Bewertungen - die zu einer neuen Bestimmung der (nur scheinbar) verloren gegangenen deutschen Identität dienen. Mit diesem Anliegen werden mentalitätsgeschichtliche Implikationen fokussiert. 
Mentalitäten, mentale Dispositionen, Denkstile, Einstellungen, Haltungen, Attitüden ${ }^{11}$ manifestieren sich als »kollektiv angeeignete Kultur « (Kuhlemann 1996: 209) in Handlungen. Indem Mentalität verstanden wird als »das Ensemble der Weisen und Inhalte des Denkens und Empfindens, das für ein bestimmtes Kollektiv in einer bestimmten Zeit prägend ist « (Dinzelbacher 1993: XXI), und indem Sprechen und Handeln in eins zu setzen sind, sind Sprachanalyse und Mentalitätsanalyse, Sprachgeschichte und Einstellungsgeschichte zwei spezifisch aufeinander bezogene Disziplinen. Es ist unbestritten, dass »Mentalitäten, Einstellungssysteme, Bewertungen, Stereotype < [...] für die sprachhistorische Analyse an Bedeutung [gewinnen] « (Mattheier 1990: 309). Für die Sprachwissenschaft liegt dementsprechend eine Definition von Fritz Hermanns vor: Mentalität ist »die Gesamtheit von Dispositionen zu einer Art des Denkens, Fühlens, Wollens - die Gesamtheit der kognitiven, affektiven (emotiven) sowie volitiven Dispositionen - einer Kollektivität«, worunter auch »Dispositionen [...] zu sprachlichem Verhalten « zu verstehen sind (Hermanns 1995: 76). Aber: Eine Konkretion, eine präzise Bestimmung dessen, was linguistische Mentalitätsanalyse ist, müssen wir noch vermissen - wiewohl wir schöne Beispiele sprachzugewandter Mentalitätsgeschichte haben, die Studie Angelika Linkes (1996) allen voran.

Um uns diese wichtige Beschreibungskategorie zu sichern, gebe ich Folgendes zu bedenken: Wenn Einstellungsgeschichte Gegenstand von Sprachgeschichte ist, kann bzw. sollte sie dies nur sein in Bezug auf eben solche Einstellungen konkret manifestierende sprachliche Objektivationen. Mentalitätsgeschichtlich orientierte Sprachwissenschaft ist auf die sprachliche Kodierung von Denken, Fühlen, Wollen und Sollen angewiesen. Dem Sprachwissenschaftler/der Sprachwissenschaftlerin ist es nur dann möglich, sinnvoll von Einstellungen zu reden, wenn ein sprachlich materialisierter Ausdruck diese repräsentiert, besser noch expliziert. Am Beispiel: Artikel 131 des Grundgesetzes weist den Gesetzgeber an, ein Bundesgesetz zu erlassen, welches die Wiedereinsetzung nazistisch belasteter Beamter in den Staatsdienst vorsieht. Der Kernsatz des Artikels lautet: "Die Rechtsverhältnisse von Personen einschließlich der Flüchtlinge und Vertriebenen, die am 8. Mai 1945 im öffentlichen Dienste standen, aus anderen als beamten- oder tarifrechtlichen Gründen ausgeschieden sind und bisher nicht oder nicht ihrer früheren

11 Versuche einer terminologischen Differenzierung wurden unternommen. Wir unterscheiden terminologisch nicht, was oftmals nicht zu unterscheiden ist. Kuhlemann (1996: 193) etwa fuhrt in diesem Sinn die drei Kategorien Totalmentalităten (»epochale, mehr oder weniger von allen Zeitgenossen geteilte Einstellungen und Selbstverständlichkeiten «), Makromentalitäten (»können [...] nationale oder auch religiöse Unterschiede innerhalb einer Epoche einfangen a) und Mikromentalitäten/Partikularmentalităten (»repräsentieren [...] zusătzliche Differenzierungen «) ein. 
Stellung entsprechend verwendet werden, sind durch Bundesgesetz zu regeln«. Von Historikern wird dieser Artikel zu Recht als Ausdruck einer in der frühen Bundesrepublik typischen Schlussstrichmentalität bewertet, so etwa von Norbert Frei, dessen Formulierungen an dieser Einschätzung keinen Zweifel lassen. ${ }^{12}$ Steht der Linguistik, die ihre Erkenntnisse aus der Analyse des sprachlichen Ausdrucks von Texten gewinnt, eine entsprechende Bewertung zu? Aus linguistischer Sicht bietet der Grundgesetzartikel 131 in seiner kühlen Nüchternheit kaum einen sprachlich manifesten Beleg zum Nachweis einer spezifischen Mentalität.

Da Mentalitäten nicht aus jeglichem textlichen Manifest linguistisch beschreibbar sind, müssen wir akzeptieren, dass unsere empirische Basis für mentalitätsspezifische Erkenntnisse um ein Vieles beschränkter ist als diejenige der weniger exakten kulturwissenschaftlichen Disziplinen. Wenn die Alliierten in den ersten Nachkriegsjahren von den besiegten Deutschen einerseits als hilfreich, freundlich, großzügig, anderseits als misstrauisch, schadensfroh und als Belastung beim Aufbau der Demokratie wahrgenommen werden, dann ist uns eine - auch anderwärts beobachtbare - mentale Schizophrenie des Denkkollektivs der intellektuellen deutschen Nachkriegsgesellschaft offenbar. Prädikationen wie diese geben uns ein mentalitätsanalytisches Placet.

Deshalb: Eine sprachlich basierte Mentalitätsgeschichte ist eigentlich und seriöserweise nur zu haben im Sinn einer Mentalitätsbezeichnungsgeschichte, die Beschreibungen eigener bzw. beobachteter Haltungen und Einstellungen zum Gegenstand hat. Wenn solche mentale Dispositionen kommentierend, problematisierend, wertend, explizierend thematisiert werden, dokumentieren sie Bewusstseinslagen, Mentalitätsbewusstsein. Die sprachhistorische Einordnung solcher Texte ist Mentalitätsbewusstseinsgeschichte. ${ }^{13}$ Erklärungsversuche der deutschen Nachkriegsintellektuellen hinsichtlich der Entstehung des Nationalsozialismus sind in diesem Sinn zu Teilen Mentalitätsbewusst-

12 »Spätestens seit 1948 [...] war in Politik und Verwaltung der Entschluß herangereift, ein [...] höchst unliebsames Element alliierter Besatzungspolitik ruckgängig zu machen, in dem Säuberungswille und Reformeifer eine aus deutscher (Beamten-)Sicht fatale Verbindung eingegangen waren: die politisch begründete Entlassung großer Teile des bei Kriegsende vorgefundenen offentlichen Dienstes, die nach den Vorstellungen von Briten und Amerikanern zugleich den Auftakt bilden sollte für eine tiefgreifende Umgestaltung des überkommenen Berufsbeamtentums $\ll$ (Frei 1996: 69).

13 Diese Bezeichnung habe ich in Analogie zu s Sprachbewusstseinsgeschichte gewählt, die bekanntlich in den letzten Jahren einige Aufmerksamkeit erfahren hat. 
seinsgeschichte, wenn sie etwa mit typisch deutschem Kadavergehorsam, Untertanengeist und Gemeinschaftswille Gründe benennen. ${ }^{14}$

\section{Fazit}

Kulturgeschichte - Diskursgeschichte - Mentalitätsgeschichte - in dieses Raster habe ich meine Überlegungen zu einer (künftigen) Linguistik eingefügt,

1. um die Notwendigkeit von Entdisziplinierung und Disziplinierung herauszustellen, die aus der Teilnahme der Sprachwissenschaft an der Superdisziplin Kulturwissenschaft resultiert;

2. um zu begründen, dass - nicht zuletzt vor dem Hintergrund der heutigen Möglichkeiten, große Textmengen zu verarbeiten - die diskursive Serie unverzichtbar ist, wenn Sprachwissenschaft dem Epitheton >kulturwissenschaftlich ' gerecht werden will;

3. um schließlich darzulegen, dass Sprachwissenschaft einen genuinen Beitrag zur Geschichte von Einstellungen leisten kann, wenn sie sich an den sprachlichen Ausdruck hält - ein Selbstverständnis von Sprachgeschichte als Mentalitätsbezeichnungsgeschichte und als Mentalitätsbewusstseinsgeschichte ist damit gerechtfertigt.

\section{Literatur}

Auer, Peter (2000): Die Linguistik auf dem Weg zur Kulturwisenschaft? - In: Freiburger Universitätsblätter. Hrsg. im Auftrag des Rektors der Albert-Ludwigs-Universität. Heft 147, März 2000. Freiburg: Rombach Verlag, 55-68.

Beaugrande, Robert de (1997): Textlinguistik: Zu neuen Ufern? - In: Gerd Antos und Heike Tietz (Hrsg.): Die Zukunft der Textlinguistik. Traditionen, Transformationen, Trends. Tubingen: Niemeyer, 1-11.

Berger, Peter L. / Luckmann, Thomas (1999): Die gesellschaftliche Konstruktion der Wirklichkeit. Eine Theorie der Wissenssoziologie. 16. Aufl. Frankfurt a.M.: Fischer.

Busse, Dietrich / Teubert, Wolfgang (1994): Ist Diskurs ein sprachwissenschaftliches Objekt? Zur Methodenfrage der historischen Semantik. - In: Dietrich Busse, Fritz Hermanns, Wolfgang Teubert (Hrsg.): Begriffsgeschichte und Diskursgeschichte.

14 Vgl. dazu Kämper (demnächst). 
Methodenfragen und Forschungsergebnisse der historischen Semantik. Opladen: Westdeutscher Verlag, 10-27.

Dinzelbacher, Peter (1993): Europäische Mentalitätsgeschichte. Hauptthemen in Einzeldarstellungen. Stuttgart: Kröner.

Ehlich, Konrad (Hrsg.) (1994): Diskursanalyse in Europa. Frankfurt a.M. [etc.]: Suhrkamp.

Fleck, Ludwik (1980): Entstehung und Entwicklung einer wissenschaftlichen Tatsache. Einführung in die Lehre vom Denkstil und Denkkollektiv. Mit einer Einleitung herausgegeben von Lothar Schäfer und Thomas Schnelle. Frankfurt a.M.: Suhrkamp [Erstausgabe 1934].

Frei, Norbert (1996): Vergangenheitspolitik. Die Anfänge der Bundesrepublik und die NS-Vergangenheit. München: Beck.

Fraas, Claudia (1996): Gebrauchswandel und Bedeutungsvarianz in Textnetzen. Die Konzepte IDENTITÄT und DEUTSCHE im Diskurs zur deutschen Einheit. Tübingen: Narr.

Hansen, Klaus P. (1990): Kultur und Kulturwissenschaft. 2., vollst. uberarb. u. erw. Aufl. Tubingen/Basel: Francke.

Hermanns, Fritz (1995): Sprachgeschichte als Mentalitätsgeschichte. Überlegungen zu Sinn und Form und Gegenstand historischer Semantik. - In: Andreas Gardt, Klaus J. Mattheier, Oskar Reichmann (Hrsg.): Sprachgeschichte des Neuhochdeutschen - Gegenstände, Methoden, Theorien. Tübingen: Niemeyer, 69-102.

Hermanns, Fritz (demnächst): »Bilder im Kopf«. Zur Wiederauferstehung des Begriffes der Idee und der Vorstellung in den Begriffen des Stereotyps, des Schemas, des frame sowie ăhnlicher Begriffe. - In: Peter Wiesinger (Hrsg.): Akten des X. Internationalen Germanistenkongresses. Frankfurt a.M. [etc.]: Lang.

Kienpointner, Manfred (1992): Alltagslogik. Struktur und Funktion von Argumentationsmustern. Stuttgart/Bad Cannstatt: Frommann-Holzboog Verlag.

Klein, Josef (1989): Wortschatz, Wortkampf, Wortfelder in der Politik. - In: Ders. (Hrsg.): Politische Semantik. Bedeutungsanalytische und sprachkritische Beiträge zur politischen Sprachverwendung. Opladen: Westdeutscher Verlag, 3-50.

Klein, Josef (2000): Komplexe topische Muster. Vom Einzeltopos zur diskurstypspezifischen Topos-Konfiguration. - In: Topik und Rhetorik. Ein interdisziplinăres Symposium. Hrsg. v. Thomas Schirren und Gert Ueding. Tübingen: Niemeyer, 623-649.

Konerding, Klaus-Peter (1993): Frames und lexikalisches Bedeutungswissen. Untersuchungen zur linguistischen Grundlegung einer Frametheorie und zu ihrer Anwendung in der Lexikographie. Tubingen: Niemeyer.

Kopperschmidt, Josef (1989): Methodik der Argumentationsanalyse. Stuttgart/Bad Cannstatt: Frommann-Holzboog Verlag.

Kuhlemann, Frank-Michael (1996): Mentalitätsgeschichte. Theoretische und methodische Überlegungen am Beispiel der Religion im 19. und 20. Jahrhundert. - In: Wolfgang Hardtwig und Hans-Ulrich Wehler (Hrsg.): Kulturgeschichte heute. Göttingen: Vandenhoeck \& Ruprecht, 182-211.

Linke, Angelika (1996): Sprachkultur und Bürgertum. Zur Mentalitătsgeschichte des 19. Jahrhunderts. Stuttgart/Weimar: Metzler. 
Mainguenau, Dominique (1994): Die >franzósische Schule< der Diskursanalyse. - In: Konrad Ehlich (Hrsg.): Diskursanalyse in Europa. Frankfurt a.M. [etc.]: Suhrkamp, 187-195.

Mattheier, Klaus J. (1990): Sprachgeschichte als Sozialgeschichte. Über eine (mögliche) Einbettung der Sprachgeschichte in die Sozialgeschichte, diskutiert an der deutschen Sprachgeschichte. - In: Dietmar Petzina und Jürgen Reulecke (Hrsg.): Bevölkerung, Wirtschaft, Gesellschaft seit der Industrialisierung. Festschrift für Wolfgang Köllmann zum 65. Geburtstag. Dortmund: Gesellschaft für Westfälische Wirtschaftsgeschichte, 293-309.

Oexle, Otto Gerhard (1996): Geschichte als Historische Kulturwissenschaft. - In: Wolfgang Hardtwig und Hans-Ulrich Wehler (Hrsg.): Kulturgeschichte heute. Göttingen: Vandenhoeck \& Ruprecht, 14-40.

Stötzel, Georg / Wengeler, Martin (1995): Kontroverse Begriffe. Geschichte des offentlichen Sprachgebrauchs in der Bundesrepublik Deutschland. Berlin/New York: de Gruyter. 